\title{
Civilisations
}

Revue internationale d'anthropologie et de sciences

humaines

$60-2 \mid 2012$

Être ou ne pas être balkanique

\section{Theo Angelopoulos}

un cinéaste grec, balkanique et universel

Marianne Mesnil et Yannis Thanassekos

\section{(2) OpenEdition}

\section{Journals}

Édition électronique

URL : http://journals.openedition.org/civilisations/2927

DOI : 10.4000/civilisations. 2927

ISSN : 2032-0442

Éditeur

Institut de sociologie de l'Université Libre de Bruxelles

\section{Édition imprimée}

Date de publication : 27 juillet 2012

Pagination : 7-10

ISSN : 0009-8140

\section{Référence électronique}

Marianne Mesnil et Yannis Thanassekos, «Theo Angelopoulos », Civilisations [En ligne], 60-2 | 2012,

mis en ligne le 30 août 2015, consulté le 01 mai 2019. URL : http://journals.openedition.org/

civilisations/2927 ; DOI : 10.4000/civilisations.2927

\section{(c) Tous droits réservés}




\title{
Hommage
}

\section{Theo Angelopoulos, un cinéaste grec, balkanique et universel}

\author{
Marianne MESNIL et Yannis THANASSEKOS
}

Ce 24 janvier 2012, disparaissait le grand cinéaste grec Theo Angelopoulos. Une très brève séquence du Journal télévisé de France 2 a relaté l'événement le soir même. Rappelant la Palme d'Or qui lui avait été décernée à Cannes en 1995, le journaliste qui a commenté la triste nouvelle a souligné, à juste titre, combien les circonstances de sa mort tragique étaient une image inversée de son œuvre et, en particulier, de son rapport au temps.

En effet, le cinéaste qui, au mépris de toute règle commerciale, sut créer dans ses films un temps de l'éternité (L'Eternité et un jour, 1998) rejoignit la sienne ce soir-là, fauché dans Athènes par un motard trop pressé.

À l'opposé de la contraction suffocante des lieux et des distances, et contre l'accélération assourdissante des vitesses, l'œuvre cinématographique d'Angelopoulos, depuis son premier grand film (La reconstitution, 1970) jusqu'à ses dernières réalisations (La Poussière du Temps, 2008), travaille inlassablement à réparer, à restaurer l'espace et le temps en tant qu'ils sont pour nous, au propre et au figuré, notre seule et exclusive demeure au monde. Toutes ses réalisations peuvent être vues comme une pédagogie qui s'obstine à nous apprendre à habiter à nouveau cette vieille demeure que nous avons quittée, désertée, il y a des lustres, pour les jouissances faciles de l'immédiateté et de l'éphémère.

Aussi, à travers ses films, le cinéaste grec nous donne à profusion du temps et de l'espace - car il faut du temps pour traverser du temps et des lieux pour arpenter l'espace ; temps lent, espace dilaté, tous deux emplis, saturés, d'expériences vécues, individuelles et collectives, de génération en génération et transmises par elles comme autant de fresques murales stockées dans les mémoires. Car c'est bien là la focale de l'œuvre : mémoire des lieux, mémoire des instants.

Certes, ces mémoires et ces expériences se rapportent à un univers circonscrit géographiquement, culturellement, politiquement et historiquement.

Grec, tout d'abord, Angelopoulos l'est tout au long de son œuvre enchâssée dans les souffrances et les illusions de son peuple. Le cinéaste en évoque sans cesse la mémoire qui, en Grèce, plus que partout ailleurs, pèse de ses longs siècles d'Histoire, tantôt grandiose et revendiquée, tantôt dérangeante et déniée. N'est-ce pas là le sens 
qu'on peut donner au lent mouvement giratoire qu'impose la caméra, autour de la baie de Salonique, à un énorme doigt de pierre, accusateur de l'Histoire (Paysage dans le brouillard, 1988) ? Ce fragment d'une statue antique, un hélicoptère vient, sous nos yeux, de le faire surgir du fond de la mer où il croupissait depuis des millénaires ${ }^{1}$.

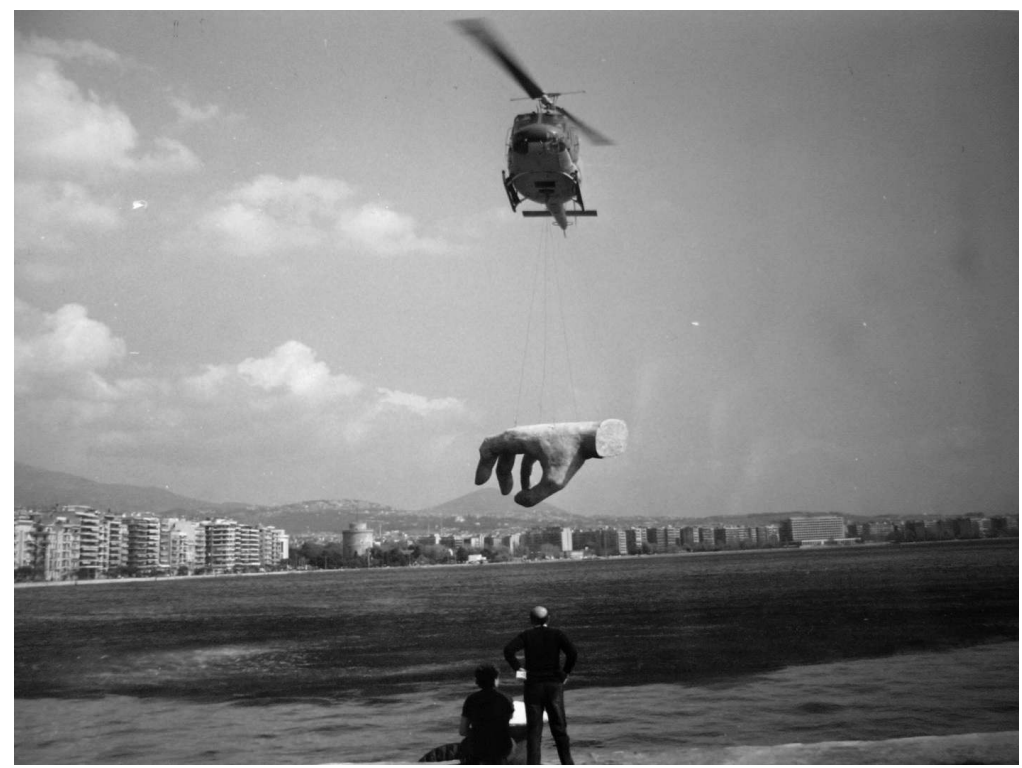

Photo de tournage du film de Teo Angelopoulos Paysage dans le brouillard (dans la Baie de Salonique).

(C) Marianna Karapiperi 1991.

La mémoire des souffrances, mais aussi et peut-être surtout, les mémoires en souffrance du peuple grec sont au cœur de la majeure partie des réalisations d'Angelopoulos. Journées de 1936 (1972) et Le voyage des comédiens (1975) - pour ne citer que ces deux films - nous donnent à voir, nous donnent à sentir, comme autant de gigantesques fresques historiques, comme autant d'insistants rappels mémoriels, les cicatrices toujours à nu laissées par ces journées, par ces années de plomb qui ont marqué l'histoire contemporaine grecque : guerre, occupations, guerre civile, répression, persécutions, arrestations, prisons, déportations, exil politique.

Dans Les Chasseurs (1977) également fait retour la mémoire encore fraîche des maquisards de la guerre civile (1946-1949)². Et c'est encore le propos de l'Apiculteur

1. Le plan est un clin d'œil d'hommage du cinéaste grec à ses collègues italiens et, en particulier, au Fellini Roma dont on se souvient du long plan de l'hélicoptère balançant au-dessus de Rome la statue de saint Pierre!

2. Le cinéaste s'est inspiré d'un fait réel : dans les années 1950, les corps de maquisards ensevelis et conservés dans la neige des hautes montagnes furent retrouvés par un groupe de chasseurs tout aussi craintifs que haineux. 
(2004), assailli par le désarroi, la solitude, le désespoir, l'étrangeté de l'existence de tous ceux qui, rescapés des années noires, peinent à survivre dans les ruines du présent.

Le cinéma d'Angelopoulos, c'est aussi la Grèce de la pauvreté qui pousse à l'émigration et vide les villages de leurs hommes, abandonnant femmes et enfants à l'attente d'un hypothétique retour. Ainsi, dès son premier long métrage ( $\mathrm{L} a$ Reconstitution 1970), le cinéaste choisit comme décor cette Grèce du nord (de l'Épire) grise, pluvieuse, hivernale; celle que ne fréquentent pas les touristes. Le drame quotidien de l'émigration pour ceux qui restent, c'est aussi le thème qui sous-tend Paysages dans le brouillard (1988), histoire de deux enfants qui veulent rejoindre leur père et s'embarquent dans un train qui les mène " vers le nord », jusqu'à ce « paysage dans le brouillard » dominé par un mirador, à l'une de ces frontières de nulle part où les petits héros du film pensent que «de l'autre côté, c'est l'Allemagne! »

Le thème des frontières, parfois meurtrières, toujours absurdes, est également un motif récurrent qu'affectionne le cinéaste. Et c'est ici que nous voyons à l'œuvre le cinéaste balkanique.

Le Pas suspendu de la cigogne (1991) et surtout le Regard d'Ulysse (1995) s'inscrivent dans un vieil espace balkanique fractionné par de " nouvelles frontières », celles des États-nations mis en place dans les grands Traités imposés par les puissances occidentales. Le pas suspendu, c'est celui d'un homme qui fait mine d'enjamber une ligne dessinée sur le sol, en narguant de piètres soldats chargés de garder les lieux. Ici encore, les miradors dominent le paysage... Cette absurdité des frontières qui séparent arbitrairement les familles, nous la retrouvons dans cette autre scène du même film où l'auteur, avec toute la force de sa poésie, fait se rejoindre un couple de jeunes mariés au milieu d'une rivière qui sépare deux terres anciennement unies.

Quant au Regard d'Ulysse, son périple s'inscrit dans une cartographie qui relève de l'ancienne Turquie d'Europe, d'Athènes à Constanta (Roumanie), en passant par Sarajevo.

Ce rapide et très incomplet parcours de la filmographie d'Angelopoulos nous montre qu'au-delà de son encrage territorial et culturel, l'œuvre a bien une portée universelle. Les thèmes et motifs de ses évocations mémorielles n'ont rien de singulier. $\mathrm{Au}$ contraire, ils touchent à cette universalité qui, depuis les temps immémoriaux, taraude l'histoire des hommes : guerres, conflits fratricides, défaites, dénuement, relégations, déplacements de populations, immigration, frontières... Toutes ces problématiques convergent finalement dans le premier volet de ce qui devait être une trilogie, le grandiose Elini (2004).

Films historiques de fiction? Films d'histoire ? En aucune façon. Films nourris d'histoire ? Certainement. Et plus encore, œuvre nourrie de mémoires, films de mémoire, films faisant mémoire. Celle des éprouvés, celle des vaincus de l'Histoire. Angelopoulos ne connaît que trop bien Clio pour se laisser prendre à son jeu. Cette fille de bonne famille, comme dirait Péguy, se contente de tourner, une par une, une fois écrites, les pages de l'Histoire, du livre de l'Histoire. Page tournée, affaire classée ! Le passé, c'est le passé ; on ne peut ni le changer, ni le modifier ; choses mortes, à jamais disparues.

Mais le talentueux cinéaste grec ne l'entend pas ainsi. Son œuvre cherche à établir, pour nous et à notre usage, un tout autre rapport avec l'Histoire, avec le passé. Pour 
y parvenir, il met à contribution l'impétueuse Mnèmè, Mnèmosynè, l'éternelle rivale de Clio. Mère des Muses, la Mémoire (Mnèmè) s'applique en effet à défaire, à déjouer en permanence le travail accompli par Clio. Elle retourne, elle parcourt les pages de l'histoire en sens inverse.

Autrement dit, s'appuyant sur Mémoire, le cinéaste n'a de cesse que de ramener le passé au présent, de le faire revivre au présent, de nous rendre contemporain ce qui, sans cela, serait irrémédiablement révolu. Tel est l'enjeu majeur de l'œuvre : rendre présent l'absent, visible l'invisible, par la résurrection des paysages, des visages, des acteurs du passé, par la résurrection rédemptrice de l'histoire. Il y a du Benjamin chez Angelopoulos ${ }^{3}$.

Impossible de passer sous silence l'importance de la musique dans l'œuvre d'Angelopoulos. Elle est en parfaite « harmonie » avec l'ensemble de son projet. La compositrice Elini Karaïndrou y a fait un travail en parfaite symbiose avec le réalisateur. On y retrouve, au niveau sonore, toutes les intentions exprimées dans ses films. Ainsi un héritage de musique grecque et balkanique (" populaire ») vient à la rencontre d'une tradition occidentale de musique symphonique pour exprimer, dans un langage sonore universel toute la gamme des sentiments de lyrisme, de drame, de nostalgie, de tragédie.

Enfin comment ne pas mentionner également la perspicacité dont ont fait preuve le cinéaste et ses collaborateurs dans le choix des lieux, des bâtiments, des paysages qui révèlent, in fine, une véritable topographie chargée d'épaisseur historique.

Le cinéma d'Angelopoulos, c'est un regard tour à tour amère, mélancolique, nostalgique, mais toujours douloureux, jeté sur les déchirures et les ruines qu'ont laissé les fureurs du passé, présence que la modernité travaille à effacer. Vu sous cet angle, son cinéma n'a jamais été aussi actuel ! Et il restera sans doute l'une des plus grandes figures du cinéma grec, balkanique et universel.

3. Nous nous référons ici au texte quasi-testamentaire de Walter Benjamin, « Sur le concept d'histoire », et singulièrement à sa troisième thèse : « Le chroniqueur, qui rapporte les événements sans distinguer entre les grands et les petits, fait droit à cette vérité : que rien de ce qui eut jamais lieu n'est perdu pour l'histoire. Certes, ce n'est qu'à l'humanité rédimée qu'échoit pleinement son passé. C'est-à-dire que pour elle seule son passé est devenu intégralement citable. Chacun des instants qu'elle a vécus devient une citation à l'ordre du jour - et ce jour est justement celui du Jugement dernier » (Euvres, t. III, Gallimard, coll. Folio, 2000, p. 429). 